\title{
Research Paper: What Is the Acute Effect of Dry Needling on the Active Trigger Points of Upper Tra- pezius Muscle? The Effect of Eliciting Local Twitch Response on Clinical Outcomes
}

\author{
Iman Kamali Hakim ${ }^{1}$, Ismail Ebrahimi Takamjani ${ }^{1 *}$, Javad Sarrafzadeh ${ }^{1}$ Kamran Ezzati $^{2}$ \\ 1. Department of Physiotherapy, School of Rehabilitation Sciences, Iran University of Medical Sciences, Tehran, Iran. \\ 2. Neuroscience Research Center, Poorsina Hospital, School of Medicine, Guilan University of Medical Sciences, Rasht, Iran.
}

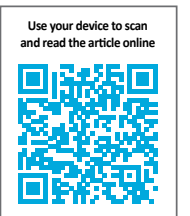

Citration: Kamali Hakim I, Ebrahimi Takamjani I, Sarrafzadeh J, Ezzati K. What Is the Acute Effect of Dry Needling on the Active Trigger Points of Upper Trapezius Muscle? The Effect of Eliciting Local Twitch Response on Clinical Outcomes. Physical Treatments. 2017; 7(1):49-57. https://doi.org/10.29252/NRIP.PTJ.7.1.49

https://doi.org/10.29252/NRIP.PTJ.7.1.49

Article info:

Received: 18 Nov. 2016

Accepted: 01 Feb. 2017

Keywords:

Myofascial trigger point syndrome, Dry needling, Local twitch response

\begin{abstract}
A B S T R A C T
Purpose: Dry needling has been introduced as an effective method to treat the upper trapezius myofascial pain. Muscle damage after receiving the local twitch response can increase the risk of tissue fibrosis in some cases. This study aimed to investigate how the clinical parameters change after dry needling without local twitch response.

Methods: This is a quasi-experimental study, with pretest and posttest. A total of 26 patients suffering from neck pain with an active trigger point in their upper trapezius muscles were recurited via the convenience sampling methods. In all patients, the needle was moved 15 times in the trigger point of the trapezius muscle and then remained in place for 5 minutes. Participants were assigned in the dry needling with local twitch response (experimental group) when a local twitch response was evoked from muscle and without receiving local twitch response or deqi (control group) when a local twitch response was not seen. Then, they were treated with one session of dry needling. Before the intervention and 24 hours after the treatment, pain, pain pressure threshold, and neck disability index were evaluated. The obtained data were analyzed by multivariate ANCOVA using SPSS version 20.
\end{abstract}

Results: After the treatment, no significant changes were seen in the experimental group compared to the control group $(\mathrm{P}>0.05)$ regarding the pain, the pain pressure threshold, and neck disability index.

Conclusion: Dry needling along with receiving local twitch response does not have a superiority over the dry needling without receiving the local twitch response while the treatment aimed to receive the immediate effects.

\footnotetext{
* Corresponding Author:

Ismail Ebrahimi Takamjani, PhD

Address: Department of Physiotherapy, School of Rehabilitation Science, Iran University of Medical Sciences, Tehran, Iran

Phone: +98 (912) 2190964

E-mail: imankamalipt@yahoo.com,driebrahimi@yahoo.com
} 


\section{Introduction}

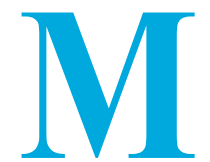

yofascial trigger point is one of the main causes of acute and chronic pain and also one of the most commonly neglected musculoskeletal problems [1]. The trigger point is a highly sensitive point within a taut musculoskeletal band that gets very painful due to pressure, strain, contraction, and overloading, and often creates the referred pain at a faraway point [1]. Simon described the main conditions of the muscular trigger points as follows: 1. Presence of a taut band in the skeletal muscle, 2. Presence of a highly sensitive point along the taut band in the skeletal muscle, 3. Creation of Local Twitch Response (LTR) when touching and pressing the trigger point, 4. Producing the referred pain in response to pressure, and 5. Continuous presence of referred pain based on a specific pattern. The myofascial pain syndrome is a major challenge both for diagnosis and treatment $[2,3]$.

Recent studies indicate the role of active trigger points in creating and maintaining local pain such as headache, backache, temporomandibular joint disorders, chronic pelvic pain syndrome, and neck pain [4]. Among the neck muscles, the upper trapezius is the most susceptible muscle that creates trigger point syndrome in the entire body due to exposure to permanent pressure and small strikes [5]. That is why timely and effective treatment is very important. Treatment of the trigger points comprises invasive and non-invasive methods. Several non-invasive methods such as stretching, massage, ischemic pressure, laser therapy, heat, acupuncture, ultrasound, and medications are used to cure myofascial pain $[6,7]$. Invasive methods include injections of Botox, corticosteroids, anesthetics as well as dry needles [7, 8].

In dry needling technique, an acupuncture needle is directed to the trigger point [9]. Many studies have proven the positive effects of dry needling [9-12]. In this method, the greatest effect occurs when a phenomenon called the LTR is created [9]. These are spinal reflexive contractions caused by stimulating sensitive sites within the trigger points, i.e. the superficial muscles such as upper trapezius [13]. In dry needling treatment associated with maximum LTR, the needle enters the local twitch response region repeatedly until the maximum responses are created. It usually takes one to two minutes for each trigger point [14]. The findings indicate that no more muscle twitch response is created in the same area after a large number of LTRs (7.4 \pm 3.9 needle entries on average). Thus, the sensitivity of the trigger point is reduced [14]. In dry needling therapies that the needle enters back and forth to the trigger point more than 15 times, the muscular fibers and neural axons of the end plates in the motor point region may get damaged. It may also disturb the complete restoration of muscular fibers, disconnect neural axons, and cause fibrosis (scar tissue) to some extent [15]. This method also has some complications, such as discomfort, local bleeding, and pain at the place of needling. About $6.8 \%$ of patients report at least one of these complications [9].

In acupuncture, needling is done to create and strengthen a special sense called "deqi" Acupuncturists believe that creating the sense of "deqi" is very important for therapeutic effects [16]. The sense of "deqi" is, in fact, painful sensation, heaviness, swelling, numbness, tingle and itching, warmth and diffusion when the needle enters the tissue [17]. The sense of "deqi" involves a bunch of neural fibers from fast myelinated $A \beta$ fibers with high thresholds to slow demyelinated fibers like $\mathrm{C}$ with a lower threshold. Thereby, it reduces the activity of the limbic system and pain $[18,19]$. Considering the importance of treating trigger point syndrome of the upper trapezius muscle in patients with neck pain, and the risks of dry needling associated with receiving LTR in the creation of tissue fibrosis, bleeding and tissue adhesion, it is necessary to adopt a treatment with minimal tissue damage. Despite the positive physiological effects of dry needling without receiving LTR, no study has yet evaluated the effect of this method on the parameters of pain, pain pressure threshold and disability after a treatment session for upper trapezius muscle. Therefore, the present research aims to address this effect.

\section{Materials and Methods}

This study was a quasi-experimental research with pretest and posttest, in which 26 patients with active trigger points of upper trapezius muscle were selected by non-random and available sampling method out of those patients referred to the Physiotherapy Clinic of the Rehabilitation Sciences Faculty by orthopedic surgeons and neurosurgeons of the hospitals affiliated to Iran University of Medical Sciences. This research was conducted from November 1st to February $14^{\text {th }}, 2016$, at the physiotherapy clinic of Faculty of Rehabilitation Sciences of Iran University of Medical Sciences.

The inclusion criteria were as follows: aged 30-50 years, body mass index less than $30 \mathrm{~kg} / \mathrm{m}^{2}$, neck pain for the past 3 months, a numerical scale of more than 30 $\mathrm{mm}$ based on a 100-mm Visual Analogue Scale (VAS) of pain [20], and the presence of trigger points in the touch, based on the Travell and Simon [21] evaluation criteria. These criteria included the presence of taut band in the posterior-medial fibers of the upper trapezius muscle 


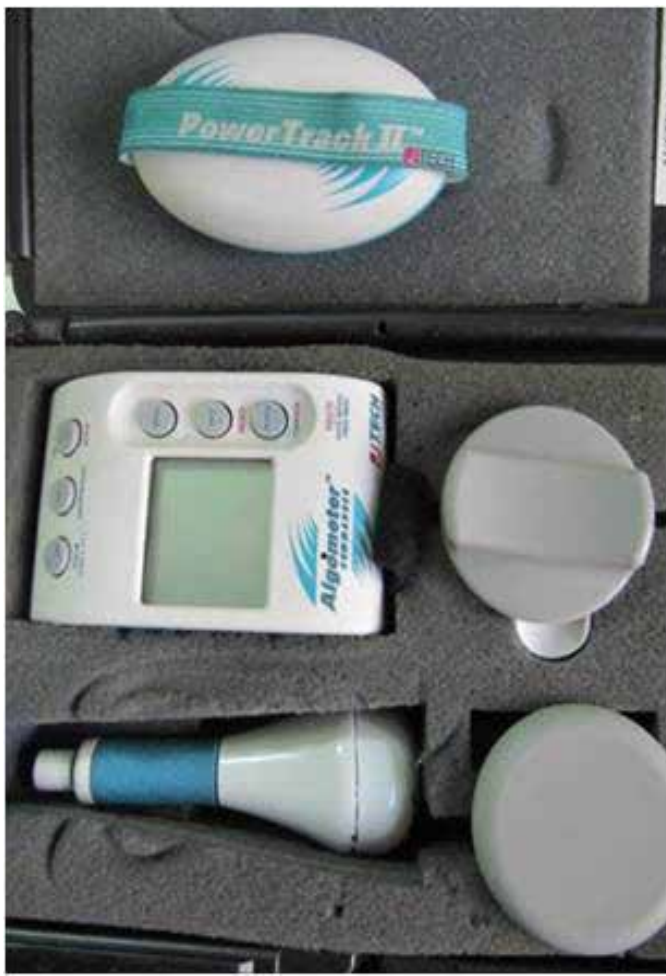

Figure 1. Digital algometer

PHYSICAL TREA MENTS

during the touch, presence of taut knot along the band, presence of at least one sensitive point in response to 25 $\mathrm{N}$ pressure, presence of referred pain and the pattern of pain associated with the trigger point of the upper trapezius muscle. The exclusion criteria included the diagnosis of fibromyalgia syndrome by taking medical history and clinical examinations based on the criteria stated by the American College of Rheumatology (1990) for not having bilateral symmetric pain in at least 11 points out of 18 (based on filling in the Fibromyalgia Impact Questionnaire), whiplash history, neck surgery in medical file and examinations, history of treatment for trigger points during past 3 months, severe postural disorders in examination, presence of prohibited conditions for dry needling including local infection, skin ulcers in the treatment area, local strike, receiving anticoagulants, or anything that may disrupt the correct assessment, such as alcohol and drug use, communication and cognitive impairment.

After entering the study, the eligible patients signed the consent forms. This study was approved by the Medical Ethics Committee of Iran University of Medical Sciences (IR.IUMS.REC.1395.0941134020). Before the evaluation and treatment, background information including participants' age, height, and weight was collected. Then, the Iranian version of the neck disability index was administrered to all patients for disability assessment. Validity and reliability of the questionnaire have been al- ready approved [22]. The questionnaire consists of 10 questions, with a minimum score of 10 and a maximum disability score of 60 . The severity of pain was also measured based on a $100-\mathrm{mm}$ VAS. All patients were asked to mark their pain on this scale. The pressure pain threshold was measured by a digital algometer with an accuracy of 0.01 (Figure 1) [22]. First, the patient was asked to sleep on the back and place the hands under the forehead. Then, the probe of algometer was placed vertically on the point and applied at a speed of $1 \mathrm{~kg} / \mathrm{s}$ to start the patient's pain and record it (3 repetitions were performed at an interval of 10 seconds, and then the average was calculated).

A digital scale was used to calibrate the instrument. After performing the evaluations, the patient was asked to sleep on the back as the hands are under the forehead. The active trigger point was detected based on the definition. In order to fix the position of the trigger points in the taut band of the posterior-medial fibers of the upper trapezius muscle, non-muscular points including spinous process of $\mathrm{C} 7$, vertebra, clavicle, and scapula process were used in the treatment session. In addition, the patients were treated with dry needling from the posterior region of the muscle to make the intervention similar for all of them (Figure 1).

Because the therapist was unaware of the creation of LTR in the muscle during the needling, the grouping was done after dry needling technique. Grouping in this study was done as follows: all patients were treated with dry needling in the first session, those with a LTR in the upper trapezius muscle were placed in the first group, and the others without LTR were placed in the second group (or the "deqi" group). The treatment method was as follows: first, the dry needling area was disinfected with the alcohol-impregnated cotton, and the examiner used sterile latex gloves. The needle used was $50 \mathrm{~mm}$ in length and $0.3 \mathrm{~mm}$ in diameter (made by Dong Bang Company in South Korea).

After detecting the location of the trigger point by touching, the thumb and index fingers of the therapist's non-dominant hand held the trigger point and then, the needle entered the skin from the side of the point by the dominant hand of the therapist. From this moment, the needle was reciprocated into the trigger point for 15 times with $1 \mathrm{~Hz}$ frequency and $1-2 \mathrm{~cm}$ along the vertical direction of the tissue [23] (without taking out the needle from the skin area). After the $15^{\text {th }}$ shot, the needle was remained in place for 5 minutes $[15,17]$. The patients were divided into two groups on the basis of the presence or absence of LTR during the reciprocating motion of the needle. In the second group (dry needling treatment group without LTR), after the evaluation and detection of 
the trigger point location by the therapist, the needle was inserted into the skin and then the trigger point. During the maneuver, the sensation of needling and the sense of "deqi" were asked from the patient. The patient's reports of "deqi" sensation were recorded $[24,25]$. The needle then remained in place for 5 minutes $[15,17]$. After 5 minutes, the needles were removed and the treatment site was disinfected again by alcohol-impregnated cotton. All patients were treated for 1 session and re-evaluated 24 hours after the treatment.

\section{Data analysis}

SPSS version 20 was used for statistical analysis. After the completion of treatment and re-evaluation of all patients, the normal distribution of data was assessed by Kolmogorov-Smirnov test. Multivariate analysis of covariance (MANCOVA) was used to compare the effect of local twitch intervention on variables and the effect of associated variables.

\section{Results}

All variables of age, weight, and height had a normal distribution $(\mathrm{P}>0.05)$. Demographic information of patients is presented in Table 1 . In addition, all patients participated in the treatment session and in both stages of evaluation. In this study, the subjects were divided into two groups based on the presence or absence of LTR, as described in the previous section (13 patients in the group of dry needling with LTR and 13 patients in the group of dry needling without LTR). In other words, 13 participants indicated no LTR when undergoing needling the upper trapezius muscle, and they were placed in the "deqi" group. In contrast, 13 patients indicated this response during dry needling in the muscle. In the following, we will discuss the changes in the parameters of these two groups.

Kolmogorov-Smirnov and Shapiro-Wilk test were used to test the normality of data and dependent variables, which is the first condition of multivariate covariance analysis (Table 2). The Levene's test was conducted to study the equality of the variables in the posttest stage, which is another condition of the multivariate covariance test. The results showed a significant level of more than 0.05 for all variables, denoting the equality of the variables (Table 3). In the next step, another condition of the multivariate covariance test was the equality of variancecovariance matrices that was performed by the Box test. The results showed no significant difference in the results of the Box test for the dependent variables $\left(\mathrm{F}_{21,2,119}=0.824\right.$ and Sig. $=0.69$; Box's M=23.854). Therefore, the assumption of equality was also confirmed.

Table 1. Demographic information of study samples

\begin{tabular}{cccc}
\hline Variable & \multicolumn{1}{c}{ Group } & Number & Mean \pm SD \\
\hline Age $(\mathrm{y})$ & With twitch response & 13 & $41.16 \pm 10.83$ \\
& Without twitch response & 13 & $46.92 \pm 9.97$ \\
Weight $(\mathrm{kg})$ & With twitch response & 13 & $77.25 \pm 14.82$ \\
& Without twitch response & 13 & $75 \pm 14.05$ \\
Height $(\mathrm{cm})$ & With twitch response & 13 & $168.5 \pm 12.96$ \\
& Without twitch response & 13 & $166.54 \pm 8.42$ \\
\hline
\end{tabular}

Table 2. Kolmogorov-Smirnov and Shapiro-Wilk test results for the normality of pretest data

\begin{tabular}{ccccccc}
\hline & & Kolmogorov-Smirnov & & Shapiro-Wilk \\
\cline { 2 - 6 } & Statistic & df & Sig. & Statistic & df & Sig. \\
\hline Pain & 0.118 & 26 & 0.2 & 0.946 & 26 & 26 \\
Pressure pain threshold & 0.179 & 26 & 0.07 & 0.872 & 0.062 & 26 \\
\hline Neck disability index & 0.091 & 26 & 0.2 & 0.977 & 0.801 \\
\hline
\end{tabular}


Table 3. Levene's test results of the equality of the variables

\begin{tabular}{ccccc}
\hline & F & df1 & df2 & Sig. \\
\hline Pain & 0.006 & 1 & 24 & 0.93 \\
Pressure pain threshold & 0.202 & 1 & 24 & 0.65 \\
Neck disability index & 0.124 & 1 & 24 & 0.72 \\
\hline
\end{tabular}

Another important assumption of the multivariate covariance test was the equality of regression coefficients. The regression coefficient equality test was investigated by the interaction of the pretest pain, pain threshold, and disability index with independent variable (treatment method) in the pretest stage. This assumption is true in relation to the posttest stage $\left(\mathrm{P}=0.64, \mathrm{~F}_{2,2441,69}=0.446\right)$. The interaction between these pretests and the independent variable was not significant, suggesting the equality of regression coefficients. Therefore, considering the confirmation of MANCOVA assumptions, we are allowed to do this test.

The relevant multivariate statistics, Wilks's lambda, were not statistically significant at $99 \%$ confidence level $(\alpha=0.01)(\mathrm{P}=0.6)$ for pain, $(\mathrm{P}=0.06)$ for sensitivity threshold and $(\mathrm{P}=0.43)$ for disability index, respectively (Table 4). Therefore, the null hypothesis was not rejected, indicating that the linear combination of the three dependent variables of posttests of pain, pressure pain threshold and neck disability index had not been affected by the independent variable (LTR of muscle) (posttest stage) after adjusting the differences between the three covariance variables (pretests of pain, pressure pain threshold and neck disability index). As a result, MANCOVA was not significant in general. In other words, the analyses show that adding LTR to dry needling of the upper trapezius muscle was not effective on the linear combination of the three variables of pain, pressure pain threshold and neck disability index.

Although the linear combination of the dependent variables was not affected by the independent variable (treatment method), to complete the covariance analysis test, this section addressed whether each dependent variable was affected by the independent variable. The comparison of the mean posttest scores is shown in Table 5. The analysis of all variables indicates that they are not individually affected by the treatment method as the independent variable.

Table 4. Results of MANCOVA, i.e. the effect of the independent variable of the treatment on the three variables after adjusting the three covariance variables at the posttest stage

\begin{tabular}{|c|c|c|c|c|c|c|}
\hline & Dependent Variable & Type III Sum of Squares & df & Sum of Squares & $\mathbf{F}$ & Sig. \\
\hline & Pain & $483.395^{\mathrm{a}}$ & 2 & 241.698 & 0.446 & 0.645 \\
\hline \multirow[t]{3}{*}{ Corrected Model } & Pressure pain threshold & $2503.440^{c}$ & 2 & 1251.720 & 3.062 & 0.066 \\
\hline & Neck disability index & $241.999^{d}$ & 2 & 120.999 & 0.874 & 0.431 \\
\hline & Pain & 6974.539 & 1 & 6974.539 & 12.879 & 0.002 \\
\hline \multirow[t]{3}{*}{ Intercept } & Pressure pain threshold & 4207.710 & 1 & 4207.710 & 10.295 & 0.004 \\
\hline & Neck disability index & 3352.089 & 1 & 3352.089 & 24.210 & 0.000 \\
\hline & Pain & 483.395 & 2 & 241.698 & 0.446 & 0.64 \\
\hline \multirow{3}{*}{$\begin{array}{l}\text { Pain } \\
\text { Pressure pain threshold } \\
\text { Neck disability index }\end{array}$} & Pressure pain threshold & 2503.440 & 2 & 1251.720 & 3.062 & 0.06 \\
\hline & Neck disability index & 241.999 & 2 & 120.999 & 0.874 & 0.43 \\
\hline & Pain & 12455.066 & 23 & 541.525 & & \\
\hline \multirow[t]{2}{*}{ Error } & Pressure pain threshold & 9400.714 & 23 & 408.727 & & \\
\hline & Neck disability index & 3184.501 & 23 & 138.457 & & \\
\hline
\end{tabular}


Table 5. Results of multivariate analysis of covariance to compare the pretest and posttest results of dependent variables in both groups

\begin{tabular}{|c|c|c|c|c|c|c|c|}
\hline & Dependent Variable & Sum of Squares & df & Mean of Squares & $\mathbf{F}$ & Sig. & Observed Power \\
\hline & Pretest & 541.337 & 1 & 541.337 & 0.986 & 0.332 & 0.158 \\
\hline \multirow[t]{2}{*}{ Pain } & Group & 215.222 & 1 & 215.222 & 0.392 & 0.538 & 0.092 \\
\hline & Error & 11529.081 & 21 & 549.004 & & & \\
\hline \multirow{3}{*}{ Pressure pain threshold } & Pretest & 184.444 & 1 & 184.444 & 0.336 & 0.568 & 0.086 \\
\hline & Group & 892.089 & 1 & 892.089 & 2.173 & 0.155 & 0.291 \\
\hline & Error & 8619.737 & 21 & 410.464 & & & \\
\hline \multirow{3}{*}{ Disability index } & Pretest & 21.238 & 1 & 21.238 & 0.039 & 0.846 & 0.054 \\
\hline & Group & 101.313 & 1 & 101.313 & 0.975 & 0.335 & 0.156 \\
\hline & Error & 2182.623 & 21 & 103.934 & & & \\
\hline
\end{tabular}

To determine if the difference between the means was so large that the results of the present study could be used to compare the two groups, the effect size was calculated by Cohen's d test [26]. The results of this test showed that the present sample size ( 26 people) had an effect size of 0.83 . Therefore, the sample size had high capacity in this study. In addition, according to the minimum detectable changes (MDC 95\%) in the variables, the following values were obtained in all variables using the formula [27]:

$$
M D C 95 \%=2.96 \times 2.25 \times S E M
$$

With the help of a 100-mm VAS for the pain variables, pressure pain threshold and neck disability index in the present study, as well as the above formula and the SEM (Standard Error of Mean) values in patients, the MDC values were $12.27,5.23$, and 5.43 for pain, pressure pain threshold and the disability index, respectively. Therefore, with respect to these values, the mean changes of the variables studied in two groups did not reach the desired level for a clinically significant difference.

\section{Discussion}

The present study aimed to examine the effect of LTR intervention in dry needling on the clinical parameters of pain, pressure pain threshold and disability in patients with active trigger points in upper trapezius muscle. As discussed in the problem statement section, considering the risks and the probability of fibrosis and bleeding in the dry needling method associated with LTR [9, 15], this study compared the effect of dry needling treatment associated with and without receiving LTR. The results indicated that the LTR did not affect pain, disability and pressure threshold of the upper trapezius myofascial. Therefore, the conventional method of dry needling associated with receiving LTR does not have a superiority over the case without receiving LTR. So, when the immediate effects of dry needling are intended, the dry needling method without receiving the LTR is the choice. The lack of significant improvement in the pressure pain threshold in the present study is consistent with previous studies in the dry needling group with LTR [28, 29]. Although pain and disability in this group showed significant improvement after 24 hours, it is similar to other studies [28, 30]. However, the results of the present study are not consistent with the results of some other researchers who did not obtain immediate effects after the dry needling method $[20,31]$.

In Irnich et al. study, a group of 36 patients with neck pain and motor deficits in the neck were treated for three sessions. Treatment sessions included a treatment session in far points, a needling session in the neck area, and a control laser session. The measurement criteria comprised pain and neck motor range. In the end, the results indicated that the needling method in the points far from the cervical myofascial involvement region was superior to the needling of the local neck area and the laser off [32]. In this study, pain and motor range did not change significantly after three sessions of dry needling. The present study indicated different results due to a different number of sessions and the second evaluation time. To explain the results, the study researchers pointed out the role of the severe muscular soreness created in dry needling technique in 
the treated muscle and the time it takes to improve the local injury. Therefore, the cause for the insignificant change in the neck's motor range seems logical in this regard. Similar results can be found in other studies.

Among other studies published in 2016, Ziaeifar et al. divided 31 patients with neck pain into two groups and treated them with one session of dry needling and spot ischemic pressure. The results showed improvement in both groups, but the difference was that the ischemic pressure treatment group showed a decrease in pressure threshold immediately after the treatment session. However, the dry needling treatment group showed pain reduction and increased pressure threshold two days after the treatment. The researchers noted the creation of muscular soreness after the dry needling as the cause of this delayed recovery. In this study method, receiving LTR was a part of the dry needling which continued until there was no more LTR [30]. The results of the present study were similar in terms of the positive effect of the needling. Despite the improvement of the patient's symptoms, the method of dry needling without LTR does not have a superiority to the other method.

In another study, Martín-Pintado-Zugasti et al. investigated the correlation between the amount of muscular soreness and gender, as well as the factors such as the number of reciprocating movements of needles in the tissue and having or lacking pain during the dry needling treatment. The evaluation included pain and pressure pain threshold three days after the treatment session. Dry needling technique was applied to upper trapezius muscle. The researchers reported that muscle soreness, pain, and pressure pain threshold was higher in women than in men. In addition, there was a strong logical correlation between the number of needles in the muscle to receive LTR and the muscle soreness, as well as pain relief and pressure pain threshold.

Therefore, the results suggest a delay effect after the dry needling technique with twitch response. There is also a relationship between the patient's gender and pain relief as well as the frequency needed to receive LTR until the end of the treatment so that there is no more LTR [33]. The study results are consistent with our study, especially in the group of dry needling without LTR. However, some results are inconsistent because the pain relief in our study was observed immediately after the dry needling technique with LTR. It can be said that the frequency of needling in this study was one of the possible causes of the difference in results. In other words, the needle entered the tissue for 15 times in the present study to complete the treatment session, while those researchers continued the needling until there was no other LTR.

There are two factors in the treatment of trigger points pain syndrome with dry needling. The first mechanism is to increase the blood flow in the surrounding area and chemical changes after the needling. The second mechanism is mechanical stimulation, which leads to the correction of the length of the sarcomeres in the affected area [34-37]. In contrast, the dry needling technique without LTR that creates the "deqi" sensation in the needle region is slightly different. In this regard, Japanese researchers have used animal studies as well as MRI imaging of the brain during needling, along with the sense of "deqi", and observed circulatory changes in the limbic system, as well as the amygdala, hippocampus, thalamus and cerebellum insula. In other words, this method of needling stimulates a batch of pain receptors from Type II to $A \beta$ and $\mathrm{C}$ and $\mathrm{A} \beta$ and thereby reduces pain $[18,19,38,39]$. Nevertheless, research into the mechanisms of dry needling effect is still ongoing in the "deqi" method.

The present study has some limitations. For example, there are no follow-up courses to examine the long-term effects of these two methods. However, no more treatment sessions were possible due to time constraints. Therefore, further research with more treatment sessions and longer follow-up is recommended. According to the study results, dry needling has a positive effect on the alleviation of pain in patients with trigger point pain syndrome of the upper trapezius muscle. Nevertheless, the needling method associated with receiving LTR does not have a superiority over the dry needling without receiving LTR. Therefore, when the immediate effects of dry needling are intended, the dry needling method without receiving the LTR can be used to treat patients due to the lower risks of bleeding and tissue fibrosis.

\section{Acknowledgements}

The present article was based on the analysis of some parts of master thesis of Iman Kamali, MSc. Student of Physiotherapy, Iran University of Medical Sciences. We appreciate the Vice-Chancellor for Research and thank the patients who participated in this study.

\section{Conflict of Interest}

The authors declared no conflicts of interest. 


\section{References}

[1] Dommerholt J. Trigger point dry needling, An evidence, and clinical-based Approach. London: Churchill Livingstone; 2013.

[2] Niel-Asher S. The concise book of trigger points. Berkeley, California: North Atlantic Books; 2008.

[3] Srbely JZ. New trends in the treatment and management of myofascial pain syndrome. Current Pain and Headache Reports. 2010; 14(5):346-52. doi: 10.1007/s11916-010-0128-4

[4] Dommerholt J, Huijbregts P. Myofascial trigger points: pathophysiology and evidence-informed diagnosis and management. Burlington, Massachusetts: Jones \& Bartlett Learning; 2010.

[5] Fernández-de-las-Peñas C, Alonso-Blanco C, Miangolarra JC. Myofascial trigger points in subjects presenting with mechanical neck pain: A blinded, controlled study. Manual Therapy. 2007; 12(1):29-33. doi: 10.1016/j.math.2006.02.002

[6] Hong CZ. Myofascial pain therapy. Journal of Musculoskeletal Pain. 2004; 12(3-4):37-43. doi: 10.1300/J094v12n03_06

[7] Melzack R. Myofascial trigger points: relation to acupuncture and mechanisms of pain. Archives of Physical Medicine and Rehabilitation. 1981; 62(3):114-7. PMID: 6972204

[8] Chen JT, Chen SM, Kuan TS, Chung KC, Hong CZ. Phentolamine effect on the spontaneous electrical activity of active loci in a myofascial trigger spot of rabbit skeletal muscle. Archives of Physical Medicine and Rehabilitation. 1998; 79(7):790-4. PMID: 9685092

[9] Kalichman L, Vulfsons S. Dry needling in the management of musculoskeletal pain. The Journal of the American Board of Family Medicine. 2010; 23(5):640-6. doi: 10.3122/jabfm.2010.05.090296

[10] Tekin L, Akarsu S, Durmuş O, Çakar E, Dinçer Ü, Kıralp MZ. The effect of dry needling in the treatment of myofascial pain syndrome: A randomized double-blinded placebo-controlled trial. Clinical Rheumatology. 2012; 32(3):309-15. doi: 10.1007/s10067-012-2112-3

[11] Tough EA, White AR, Cummings TM, Richards SH, Campbell JL. Acupuncture and dry needling in the management of myofascial trigger point pain: A systematic review and metaanalysis of randomised controlled trials. European Journal of Pain. 2009; 13(1):3-10. doi: 10.1016/j.ejpain.2008.02.006

[12] Tsai CT, Hsieh LF, Kuan TS, Kao MJ, Chou LW, Hong CZ. Remote effects of dry needling on the irritability of the myofascial trigger point in the upper trapezius muscle. American Journal of Physical Medicine \& Rehabilitation. 89(2):133-40. doi: 10.1097/phm.0b013e3181a5b1bc

[13] Rha D, Shin JC, Kim YK, Jung JH, Kim YU, LeeSC. Detecting local twitch responses of myofascial trigger points in the lower-back muscles using ultrasonography. Archives of Physical Medicine and Rehabilitation. 2011; 92(10):1576-1580.e1. doi: 10.1016/j.apmr.2011.05.005

[14] Hsieh YL, Kao MJ, Kuan TS, Chen SM, Chen JT, Hong CZ. Dry needling to a key myofascial trigger point may reduce the irritability of satellite MTrPs. American Journal of Physical Medicine \& Rehabilitation. 2007; 86(5):397-403. doi 10.1097/phm.0b013e31804a554d
[15] Domingo A, Mayoral O, Monterde S, Santafé MM. Neuromuscular damage and repair after dry needling in mice. evidence-based complementary and alternative medicine. Hindawi Limited; 2013; 2013:1-10. doi: 10.1155/2013/260806

[16] Shankar H, Reddy S. Two- and three-dimensional ultrasound imaging to facilitate detection and targeting of taut bands in myofascial pain syndrome. Pain Medicine. 2012; 13(7):971-5. doi: 10.1111/j.1526-4637.2012.01411.x

[17] Rickards LD. Therapeutic needling in osteopathic practice An evidence-informed perspective. International Journal of Osteopathic Medicine. 2009; 12(1):2-13. doi: 10.1016/j. ijosm.2009.01.003

[18] Lu GW. Neurobiologic research on acupuncture in china as exemplified by acupuncture analgesia. Anesthesia \& Analgesia. 1983; 62(3):335-40. doi: 10.1213/00000539-19830300000009

[19] Lu G. Characteristics of afferent fiber innervation on acupuncture points zusanli. American Journal of PhysiologyRegulatory, Integrative and Comparative Physiology. 1983; 245(4):606-12. PMID: 6624955

[20] Rayegani SM, Bayat M, Bahrami MH, Elyaspour D, Azhar A, Valaei N. [Comparison of dry needling and physical therapy modalities in the treatment of myofascial pain of upper trapezius muscle (Persian)]. Research in Medicine; 34(3):15763.

[21] Simons DG, Travell JG, Simons LS. Travell \& Simons' myofascial pain and dysfunction: Upper half of body. Philadelphia: Lippincott Williams \& Wilkins; 1999

[22] Mousavi SJ, Parnianpour M, Montazeri A, Mehdian H, Karimi A, Abedi M, et al. Translation and validation study of the Iranian versions of the neck disability index and the neck pain and disability scale. Spine. 2007; 32(26):825-31. doi: 10.1097/brs.0b013e31815ce6dd

[23] Dommerholt J, Mayoral del Moral O, Gröbli C. Trigger point dry needling. Journal of Manual \& Manipulative Therapy. 2006; 14(4):70-87. doi: 10.1179/jmt.2006.14.4.70e

[24] Cagnie B, Barbe T, De Ridder E, Van Oosterwijck J, Cools A, Danneels L. The influence of dry needling of the trapezius muscle on muscle blood flow and oxygenation. Journal of Manipulative and Physiological Therapeutics. 2012 35(9):685-91. doi: 10.1016/j.jmpt.2012.10.005

[25] Nabeta T, Kawakita K. Relief of chronic neck and shoulder pain by manual acupuncture to tender points - a sham-controlled randomized trial. Complementary Therapies in Medicine. 2002; 10(4):217-22. doi: 10.1016/s0965-2299(02)00082-1

[26] Rosenthal R, Rosnow RL, Rubin DB. Contrasts and effect sizes in behavioral research: A correlational approach. Cambridge: Cambridge University Press; 2000.

[27] Hidalgo B, Gilliaux M, Poncin W, Detrembleur C. Reliability and validity of a kinematic spine model during active trunk movement in healthy subjects and patients with chronic non-specific low back pain. Journal of Rehabilitation Medicine. Acta Dermato-Venereologica; 2012; 44(9):756-63. doi 10.2340/16501977-1015

[28] Ebrahimi R, Taheri N. [Pain, pressure pain threshold and disability following one session of dry needling in subjects with active trigger points in the upper trapezius muscle (Per- 
sian)]. Journal of Research in Rehabilitation Sciences. 2016; 12(2):76-81.

[29] Govender M. The effect of heat therapy on post-dry needling soreness in the deltoid muscle of asymptomatic subjects [MSc. thesis]. Durban: Durban University of Technology

[30] Ziaeifar M, Arab AM, Nourbakhsh MR. Clinical effectiveness of dry needling immediately after application on myofascial trigger point in upper trapezius muscle. Journal of Chiropractic Medicine. 2016; 15(4):252-8. doi: 10.1016/j. jcm.2016.08.009

[31] Rayegani SM, Bayat M, Bahrami MH, Raeissadat SA, Kargozar E. Comparison of dry needling and physiotherapy in treatment of myofascial pain syndrome. Clinical Rheumatology. 2013; 33(6):859-64. doi: 10.1007/s10067-013-2448-3

[32] Irnich D, Behrens N, Gleditsch JM, Stör W, Schreiber MA, Schöps P, et al. Immediate effects of dry needling and acupuncture at distant points in chronic neck pain: results of a randomized, double-blind, sham-controlled crossover trial. Pain. 2002; 99(1):83-9. doi: 10.1016/s0304-3959(02)00062-3

[33] Martín-Pintado-Zugasti A， Rodríguez-Fernández ÁL, Fernandez-Carnero J. Postneedling soreness after deep dry needling of a latent myofascial trigger point in the upper trapezius muscle: Characteristics, sex differences and associated factors. Journal of Back and Musculoskeletal Rehabilitation. 2016; 29(2):301-8. doi: 10.3233/bmr-150630

[34] Gerber LH, Shah J, Rosenberger W, Armstrong K, Turo D, Otto $\mathrm{P}$, et al. Dry needling alters trigger points in the upper trapezius muscle and reduces pain in subjects with chronic myofascial pain. PM\&R. 2015; 7(7):711-8. doi: 10.1016/j. pmrj.2015.01.020

[35] Shah JP. Uncovering the biochemical milieu of myofascial trigger points using in vivo microdialysis. Journal of Musculoskeletal Pain. 2008; 16(1-2):17-20. doi: 10.1080/10582450801960099

[36] Shah JP, Danoff JV, Desai MJ, Parikh S, Nakamura LY, Phillips TM, et al. Biochemicals associated with pain and inflammation are elevated in sites near to and remote from active myofascial trigger points. Archives of Physical Medicine and Rehabilitation. 2008; 89(1):16-23. doi: 10.1016/j. apmr.2007.10.018

[37] Shah JP, Gilliams EA. Uncovering the biochemical milieu of myofascial trigger points using in vivo microdialysis: An application of muscle pain concepts to myofascial pain syndrome. Journal of Bodywork and Movement Therapies. 2008; 12(4):371-84. doi: 10.1016/j.jbmt.2008.06.006

[38] Hui KK, Nixon EE, Vangel MG, Liu J, Marina O, Napadow $\mathrm{V}$, et al. Characterization of the "deqi" response in acupuncture. BMC Complementary and Alternative Medicine. 2007; 7(1). doi: 10.1186/1472-6882-7-33

[39] Asghar AU, Green G, Lythgoe MF, Lewith G, MacPherson $\mathrm{H}$. Acupuncture needling sensation: The neural correlates of deqi using fMRI. Brain Research. 2010; 1315:111-8. doi: 10.1016/j.brainres.2009.12.019 